Amer. J. Bot, 56(10): 1152-1159. 1969.

\title{
THE RELATION BE'TWEEN GROWTH, CONIDIATION AND TREHALASE ACTIVITY IN NEUROSPORA CRASSA ${ }^{1}$
}

\author{
David L. Hanks² and Alfred S. Sussman \\ Department of Botany, University of Michigan, Ann Arbor
}

A $\quad$ B $\mathbf{S}$ T $\mathbf{R}$ A $\quad$ C $\mathbf{T}$

\begin{abstract}
The extent to which trehalose is accumulated in the vegetative mycelium of strains of Neurospora crassa is significantly affected by conidiation. In heavily conidiating strains a rapid decrease in mycelial trehalose occurs following the initiation of conidiation. Meanwhile, trehalase activity in the vegetative mycelium of heavily conidiating strains increases rapidly following the initiation of conidiation, although apparently it is not directly caused by the sporulation process. High levels of both trehalase and trehalose appear concomitantly in the newly formed conidia.
\end{abstract}

MANY OBSERVATIONS attest to the fact that changes in the nutrient level affect the sporulation of fungi and other microorganisms (Hawker, 1957; Halvorson, 1962). Therefore, we began studies with Neurospora in which the changes in hydrolytic enzymes involved in sugar metabolism including trehalase and invertase were studied in relation to conidiation. That the activity of these enzymes in Neurospora possibly was influenced by the level of nutrients and related to conidium formation was suggested by the work of Hill and Sussman (1964). Thus the germination of conidia was accompanied by the rapid diminution of the activity of these two enzymes, and upon the leveling off of growth and the formation of conidia increased activity became evident. The ubiquity of trehalose in the fungi and its prominent role as an endogenous source of energy suggested that there might be a connection between the formation of conidia and the synthesis of trehalase, which in turn might be regulated by the supply of exogenous sugar. The results of these studies are presented in two papers, the first devoted to the relation between trehalase and conidiation and the second to the means through which trehalase synthesis is controlled.

Methods AND Materials-Strain 69-1113A $\left(\mathrm{T}^{\mathrm{s}}\right)$ of Neurospora crassa was the standard strain with which most of the experiments were performed. Another wild-type strain, 73a, was used occasionally to supplement the data obtained with strain $69-1113 \mathrm{~A}$.

Mutant strains used are listed in Table 1. With the exception of strains UM107A and B2A which were obtained from Dr. R. H. Davis,

\footnotetext{
${ }^{1}$ Received for publication 25 March 1969.

This study was supported by a N. I. H. Predoctoral Fellowship and constitutes a portion of the thesis submitted as a requirement for the $\mathrm{Ph} . \mathrm{D}$. degree of the primary author.

2 Present address: Department of Science, Northeast Missouri State College, Kirksville, Mo.
}

University of Michigan, Ann Arbor, mutant strains were obtained from the Fungal Genetics Stock Center, Department of Biological Sciences, Dartmouth College, Hanover, N. H.

Standing cultures were grown in $30 \mathrm{ml}$ of a liquid minimal medium containing $2 \%$ sucrose and $2 \%$ Vogel's minimal supplement (Vogel, 1956) in 125-ml Erlenmeyer flasks at 22-24 C. Continuous light was provided by two $\mathrm{F} 48 \mathrm{~T} 12 / \mathrm{CW}$ fluorescent tubes (General Electric) set at $4 \frac{1}{2}$ inches above the tops of the flasks. These conditions are subsequently referred to as standard growth conditions.

Inoculum for aconidial and slowly conidiating strains was prepared by homogenizing mycelial mats of approximately $100 \mathrm{mg}$ dry weight in a sterile Waring Blendor for $1 \mathrm{~min}$ in $100 \mathrm{ml}$ sterile distilled water. This preparation remained viable for several days when stored at $4 \mathrm{C}$. Conidial suspensions were used as inoculum for the other strains.

Cultures were harvested in duplicate. Young, or slow-growing cultures were separated from the growth medium by filtration through a Büchner funnel, washed several times with distilled water, dried, and weighed. Larger mycelial mats were first placed in approximately 1 liter of distilled water before the final washing and drying in a Büchner funnel. When conidia and aerial hyphae were present, they were scraped from the mycelial mat with a spatula and discarded except when the dry weight of the entire culture was desired.

The early stages of conidiation are often ac. companied by the production of large amounts of aerial hyphae bearing very few conidia. These may be readily harvested by lightly scraping the surface of the mycelium or side of the flask with a spatula. As the conidia begin to appear, a tuft of aerial hyphae may be removed from the flask with forceps, placed on paper and the portion bearing the conidia cut off with a razor blade and discarded. As more conidia are pro- 
TAHLE 1. Description of mutants of Neurospora crassa

\begin{tabular}{|c|c|c|c|c|}
\hline Locus & Allele no. & $\begin{array}{c}\text { Mating } \\
\text { type }\end{array}$ & $\begin{array}{l}\text { Linkage } \\
\text { group }\end{array}$ & F.G.S.C. no. ${ }^{\mathrm{a}}$ \\
\hline ros (inositol-less) & 89601 & $\mathbf{A}$ & VR & 497 \\
\hline pe (peach) & Y8743m & $\mathbf{A}$ & IIR & 37 \\
\hline fls (fluffyish) & STL6 & $\mathbf{A}$ & IR & 2255 \\
\hline fls, aur (fluffyish-aurescent) & STL6,34508 & $\mathbf{a}$ & IR & 60 \\
\hline os (osmotic) & E-11200 & a & IR & 34 \\
\hline sc (scumbo) & 5801 & $\mathbf{a}$ & IIIR & 115 \\
\hline fl (fluffy) & $\mathrm{L}$ & $\mathbf{A}$ & IIR & 45 \\
\hline fld (fluffyoid) & P628 & a & IVR & 115 \\
\hline sk (skin) & B106 & a & VIIR & 276 \\
\hline $\arg -12$ (arginine-requiring) & UM107 & A & - & 979 \\
\hline pan-2 (pantothenate-requiring) & B2 & $\mathrm{A}$ & VIR & 466 \\
\hline
\end{tabular}

a Fungal Genetics Stock Center, Department of Biological Sciences, Dartmouth College, Hanover, N.H.

duced, it becomes more difficult to find aerial hyphae free of spores. Organic solvents, commonly used to remove conidia from aerial hyphae, were not used since their use resulted in a loss in dry weight. The loss in weight was significant since trehalase activity was determined on the basis of dry weight.

The number of conidia produced were quantitatively determined by withdrawing the medium from the flask and then spraying the conidiating surface with carbon tetrachloride by means of a hypodermic syringe. This freed the conidia from the aerial hyphae and brought them into suspension. The conidial suspension was withdrawn from the flask, diluted to a readable range with additional sarbon tetrachloride, and counted by means of a hemocytometer.

For trehalose determinations conidia were withdrawn from the culture by means of a Pasteur pipette attached to an aspirator. A tube containing a loose glass-wool plug served as a filter to retain the fragments of aerial hyphae. This tube was connected to a $250-\mathrm{ml}$ suction flask attached to an aspirator and containing approximately 100 -ml of distilled water which acted as a trap to retain the conidia. Conidia were removed from the suspension by filtration through a Millipore filter and dried.

A second method of harvesting conidia was used in connection with the trehalase assay. After removal of the medium from the culture flask, approximately $20-30 \mathrm{ml}$ of distilled water were added and the contents swirled to bring as many conidia into suspension as possible. The conidial suspension was then filtered through a loose glasswool plug in the bottom of a Büchner funnel to remove the majority of the aerial hyphae. Conidia were removed from this suspension by centrifugation at 1,500-2,000 $\times g$ in a clinical centrifuge for $5 \mathrm{~min}$, after which all except approximately 1 $\mathrm{ml}$ of the water was decanted. The conidia were then resuspended in the remaining water and poured, drop by drop, onto the center of filter paper inserted in a Büchner funnel and dried.
For trehalose determinations, mycelial pads and conidia were placed immediately in a $100 \mathrm{C}$ oven and allowed to remain until a constant dry weight was attained.

Dry weights for the trehalase analyses were obtained by drawing approximately $50 \mathrm{ml}$ of reagent grade acetone through the mycelial mat or conidia while still in the funnel immediately following the final wash in water. An accurate dry weight can be obtained within 1-5 min following this treatment.

For trehalose determinations, the oven-dried materials were pulverized with a mortar and pestle in anhydrous ethyl ether (approximately $5 \mathrm{ml}$ per mg dry weight) and allowed to stand $24 \mathrm{hr}$ to remove most of the fatty substances. The ether extract was removed by filtration through Whatman \#1 filter paper using a Büchner funnel. Care was taken to use only the center portion of the filter so that all of the residue remained on the paper. The residue and filter paper were suspended in $50 \mathrm{ml}$ of $80 \%$ ethanol and heated to $60 \mathrm{C}$ for $30 \mathrm{~min}$, or until all of the ethanolsoluble carbohydrates were removed from the tissue. The residue was washed twice with additional $80 \%$ ethanol and discarded. The filtrate was concentrated under vacuum using a Rinco flash evaporator, model \#1007-4 IN, and the volume adjusted to $20 \mathrm{ml}$ by the addition of 0.05 M sodium phosphate buffer, $p \mathrm{H} 5.6$, hereafter referred to as standard buffer.

To duplicate 0.5 -ml aliquots of the carbohydrate extract (containing from $10-1,000 \mathrm{~g}$ trehalose), $1.4 \mathrm{ml}$ of standard buffer and $0.1 \mathrm{ml}$ of purified trehalase (Hill and Sussman, 1963) were added. The resulting mixture was incubated for $1 \mathrm{hr}$ at $37 \mathrm{C}$, which was adequate to insure complete hydrolysis of the trehalose. Reducing sugar (shown to be glucose) was quantitatively determined by the Nelson modification of the Somogyi method (Nelson, 1944). Readings were made with a Klett-Summerson colorimeter containing a \#54 filter.

For trehalase determinations, cells of acetone- 


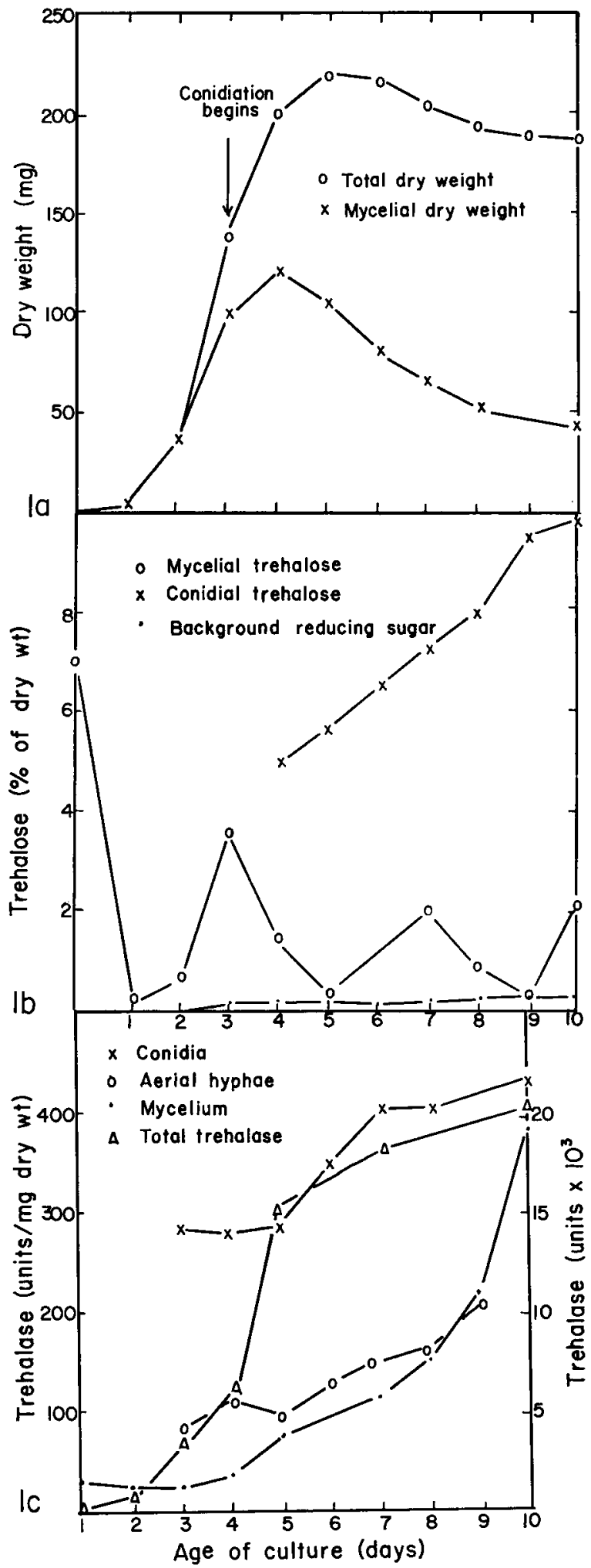

Fig. 1a-c.-Fig. 1a. Growth curve of strain 69-1113A.Fig. 1b. Trehalose accumulation in the mycelium and conidia of strain 69-1113A.-Fig. 1c. Trehalase activity in the vegetative mycelium, aerial hyphae and conidia of strain $69-1113 \mathrm{~A}$. dried materials were broken with a mortar and pestle over ice using washed and ignited sand in standard buffer solution. Extracts were either used immediately or frozen and stored at $-15 \mathrm{C}$.

Trehalase activity was determined using the following modification of the method of Hill and Sussman (1963). The majority of the enzyme can be brought into solution by extraction in standard buffer, but a significant a mount remains attached to the cell wall (Hill and Sussman, 1964). Attempts to free this fraction by extraction in $0.05 \mathrm{M}$ sodium phosphate buffer ranging in $p \mathrm{H}$ from

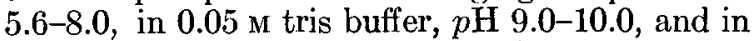
organic solvents were futile. The latter were effective in removing a large percentage of the bound protein from the wall material but had little effect on trehalase. For this reason the wall fraction was allowed to remain in the enzyme extract and the activity was calculated on the basis of dry weight. This technique yielded excellent replication.

Enzyme extract $(2.0 \mathrm{ml})$ and $0.5 \mathrm{ml}$ of a $0.5 \%$ trehalose solution were added to duplicate $15 \times 150-\mathrm{mm}$ test tubes containing $1.3 \mathrm{ml}$ standard buffer. Incubation was carried out at $37 \mathrm{C}$ for $30 \mathrm{~min}$, after which the reaction was halted by placing the tubes in a boiling water bath for $5 \mathrm{~min}$. The appearance of reducing sugar, shown to be glucose by the method of Rowher, Henschel, and Engel (1965), was determined by the Nelson modification of the Somogyi method (Nelson, 1944). Solutions were clarified by centrifugation at $1,500-2,000 \times g$ for $4 \mathrm{~min}$ just prior to taking readings for which a KlettSummerson colorimeter with a \#54 filter was used. Activity per unit weight is expressed as $\mu \mathrm{g}$ of glucose produced per mg dry weight of material. One unit of activity is defined as that amount of enzyme which produced $1 \mu \mathrm{g}$ of glucose in 30 $\min$ at $37 \mathrm{C}$.

Results-The relation between growth and the trehalose content of mycelium and conidia of strain 69-1113A, which conidiates heavily, was studied by periodic analyses of the sugar after inoculation of the culture. As Fig. 1a discloses, the maximum weight of mycelium is obtained on the 4th day of growth, after which a rapid decline occurs. However, the total weight, including conidia, continues to increase until the 5 th day and decreases only slightly thereafter. Inasmuch as conidiation begins on the $3 \mathrm{rd}$ day and there is profuse growth of aerial hyphae upon which conidia are produced during at least the next 2 days, spore formation probably accounts for the increase in total weight as that of the mycelium decreases. The growth curves of another heavily conidiating strain, 89601A (Fig. $2 a)$, closely resemble those of $69-1113 \mathrm{~A}$ in that mycelial growth ceases soon after the start of conidiation and in the other respects mentioned above. 


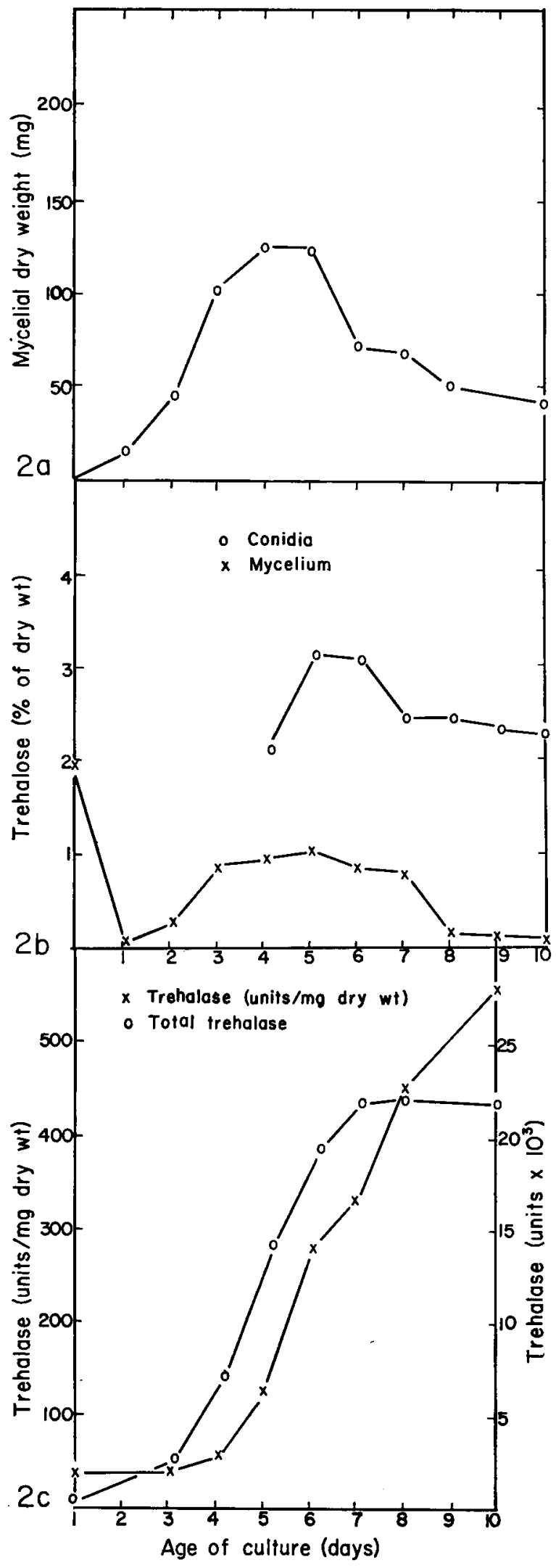

Figure $1 \mathrm{~b}$ reveals that the trehalose content of the mycelium of strain $69-1113 \mathrm{~A}$ reaches a maximum of the 3rd day of growth at the time when conidation begins but 1 day prior to the attainment of the peak in mycelial weight. After the 3rd day trehalose disappears from the mycelium, but it reappears in a reproducible cyclic fashion during the 10 days of measurement. In strain $89601 \mathrm{~A}$ (Fig. 2b) the trehalose concentration levels off in the mycelium but does not decrease as in strain 69-1113A. However, in both strains the conidia contain more trehalose than the mycelium and in those of strain $69-1113 \mathrm{~A}$ the amount continues to increase, reaching $10 \%$ by the 10 th day.

In aconidial strains STL6, 34508a, and STL6A trehalose increases in amount until growth stops (Fig. 3a).

Trehalase activity in the mycelium of strain 69-1113A remains low and rather constant during the first 3 days of growth (Fig. 1c) and increases slightly during the 4 th day and more rapidly after the 5th. In spite of the loss in mycelial weight the total trehalase activity continues to

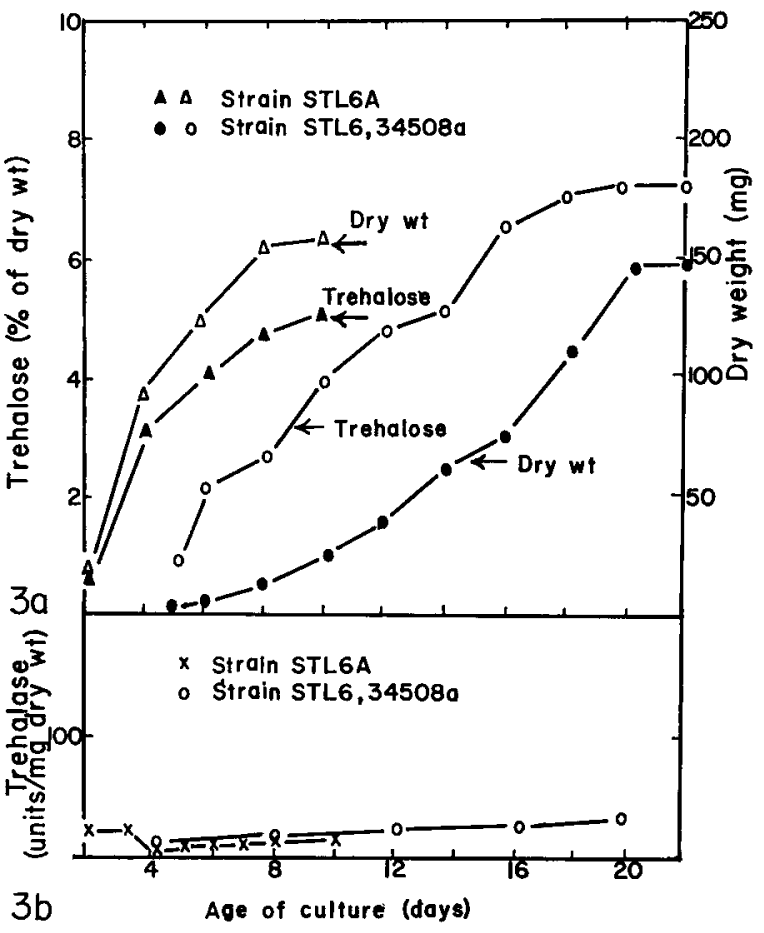

Fig. 3a-b.-Fig. 3a. Mycelial trehalose accumulation in strains STL6, 34508a and STL6A.-Fig. 3b. Mycelial trehalase activity of strains STL6, 34508a and STL6A.

Fig. 2a-c.--Fig. 2a. Mycelial growth curve of heavily conidiating strain $89601 \mathrm{~A}$.-Fig. 2b. Trehalose accumulation in the mycelium and conidia of strain 89601A.Fig. 2c. Trehalase activity in the mycelium of strain 89601A. 
increase after the peak in growth is reached. Conidial trehalase activity is approximately 10fold greater than that in the mycelium and increases as the culture ages, as does the trehalose content. Aerial hyphae, upon which the conidia are borne, show activity intermediate between that of conidia and mycelium until the 8th day after which it is not much different from that in the vegetative mycelium. The trehalase activity in the mycelium of the second heavily conidiating strain, 89601A (Fig. 2c), parallels that of strain 69-1113A.

By contrast, aconidial strains STL6A and STL6, 34501a, B106a and 5801a (Fig. 3b) have very little trehalase activity throughout their growth. Moreover, the trehalase activity in poorly conidiating strains, IA, P628A and E11200a, remains low until the latter part of the growth period after which a small increase is noted. Thus, in the cases studied the highest trehalase activities are obtained in heavily conidiating strains, with intermediate or low levels in poor conidiators and virtually none in aconidial ones.

The specificity of the relationship between conidiation and trehalase was tested by studying other enzymes, including $\beta$-galactosidase, alkaline phosphatase, ornithine transcarbamylase, tryptophan synthetase and invertase. Comparisons were made by computing the ratio of activity in the mycelium of the heavily conidiating strain, $69-1113 \mathrm{~A}$, to that in the aconidial strains, STL6A and B106a, at similar ages. This ratio for trehalase is 0.91 in 2-day-old cultures of strains 69-1113A and STL6A prior to conidiation and it rises to 26.0 by the 10th day (Table 2). By contrast the ratios of the other enzyme activities are much less affected by conidiation. An even more striking contrast is found when strain B106a is used; as in the previous case relatively small changes accompany conidiation except in the case of trehalose.

In order to test the correlation between trehalase activity and conidiation, enzyme levels were determined when the formation of spores was suppressed. This was accomplished with strain 69 $1113 \mathrm{~A}$ by eliminating $\mathrm{CaCl}_{2}$ from the standard growth medium and by the use of Turian's "M" medium (Turian, 1964). In both instances as the data in Fig. 4a reveal, trehalase activity remained low until the end of the growth period, even though the weight of the cultures is not much less than that obtained in standard media. However, when conidiation is suppressed by growing strain 69-1113A in shaken cultures, trehalase activity begins to increase during the 4th day following which the rise in activity is greater than in standing cultures (Fig. 4b).

The significance of the formation of trehalase was examined further in cultures of strain $Y 843 \mathrm{~mA}$ in which better synchrony of conidiation was possible than in the other strains used. First, cultures were grown for $24 \mathrm{hr}$ on a rotary shaker to delay conidiation which usually begins in this strain as soon as the first mycelial strands are formed. Following this, the cultures were placed at $38 \mathrm{C}$ for $4 \mathrm{hr}$, after which they were incubated at room temperature under standard growth conditions. Conidia are developing by the time the cultures are removed to room temperature and $3 \mathrm{hr}$ later sufficient are present to count. This experiment reveals that trehalase activity remains low until about $1.5 \times 10^{7}$ conidia are

TABLE 2. Ratios of specific activity of enzymes from wild-type strain 69-1113A to those in the aconidial mutants, strains STL6A and B106a

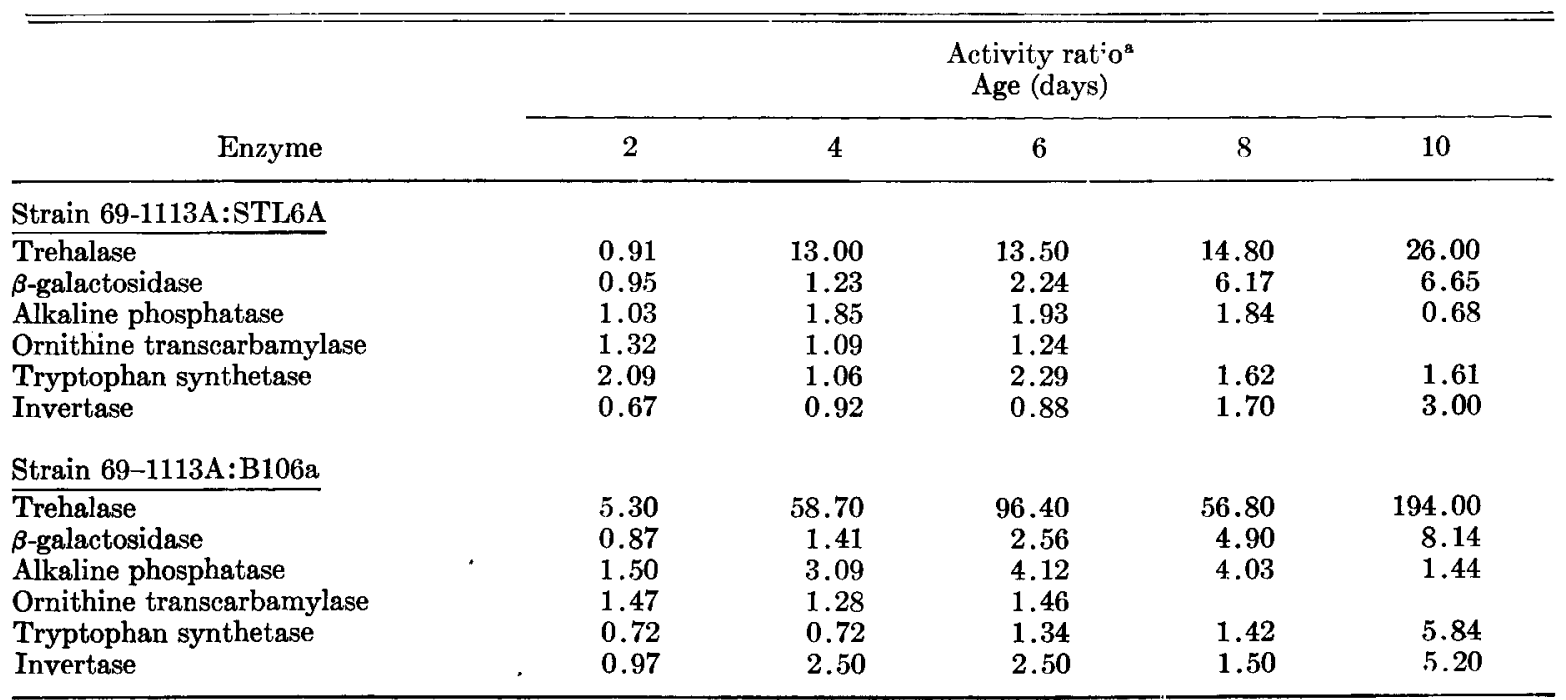

s The activity ratio is the ratio of the specific activity of enzymes from strain $69-1113 \mathrm{~A}$ to that from aconidial mutants. 


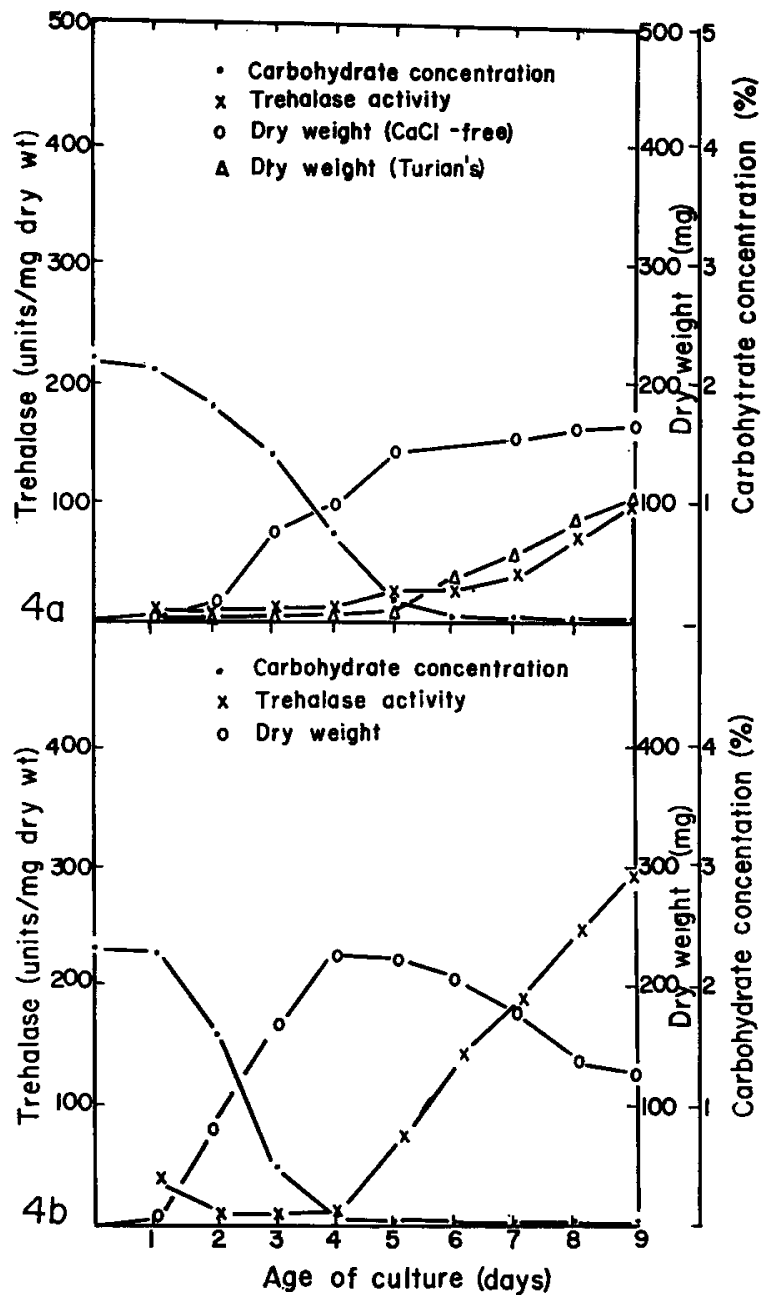

Fig. 4a-b.-Fig. 4a. Correlation of trehalase activity per unit weight, dry mycelial weight and carbohydrate concentration in the growth medium of strain 69-1113A grown in calcium chloride-free medium and Turian's conidial suppressing medium. Trehalase activity and carbohydrate concentration represent that found in chloride-free medium but is similar to that occurring in Turian's medium.-Fig. 4b. Correlation of trehalase activity, dry mycelial weight and carbohydrate concentration in the growth medium of shaken cultures of strain $69-1113 \mathrm{~A}$.

produced, after which a small increase is observed (Fig. 5a).

Furthermore, the onset of the increase in trehalase activity is delayed when strain $69-1113 \mathrm{~A}$ is grown in $6 \%$ sucrose without any apparent effect upon the time of initiation or the rate of conidiation (Fig. 5b). Thus, conidiation begins as usual during the 3rd day; by the end of the 4th day more than $3 \times 10^{9}$ conidia are formed, but trehalase activity does not begin to increase until the 5th day.

The relation between conidiation and the carbohydrate content of the growth medium is shown in Table 3 which reveals that conidia appear at the same time even though the sucrose content is varied from 1-8\%. Likewise, the substitution of glucose, fructose or trehalose for sucrose has little, if any, effect upon conidiation. Furthermore, as can be seen in Fig. 6, conidiation in strain 69$1113 \mathrm{~A}$ is initiated in the presence of more than $1 \%$ carbohydrate in the medium. A surprising aspect of these experiments is the fact that growth of the mycelium ceases abruptly on the
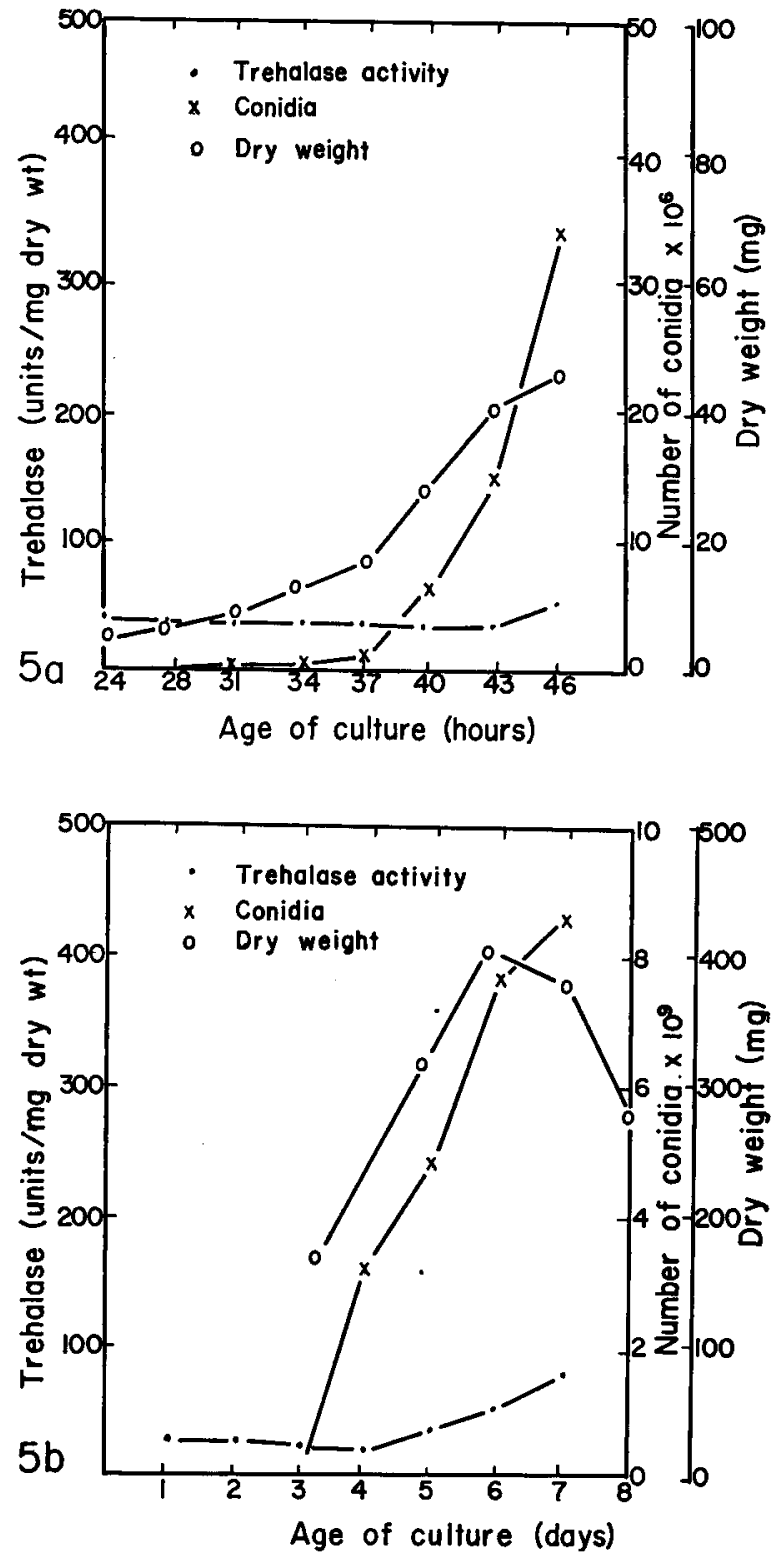

Fig. 5a-b.--Fig. 5a. Correlation between conidial production and increased levels of mycelial trehalase activity using strain Y843mA.-Fig. 5b. Correlation between eonidial production and increased levels of mycelial trehalase activity using strain $69-1113 \mathrm{~A}$ grown in medium containing $6 \%$ sucrose. 
TABLE 3. Effect of sucrose concentration upon conidiation in cultures of strain 69-1113A

\begin{tabular}{cc}
\hline $\begin{array}{c}\text { Initial } \\
\text { sucrose level } \\
(\%)\end{array}$ & $\begin{array}{c}\text { Carbohydrate } \\
\text { present when } \\
\text { conidiation begins } \\
(\%)\end{array}$ \\
\hline 1.0 & 0.35 \\
2.0 & 1.33 \\
4.0 & 3.55 \\
6.0 & 5.44 \\
8.0 & 7.33 \\
\hline
\end{tabular}

a Conidiation began in all cultures during the 3rd day when aliquots of the medium were removed and the carbohydrate content was determined with Dreywood's anthrone reagent.

4th day even though approximately $0.5 \%$ sugar still remains in the medium. By contrast, when conidiation is suppressed in this strain, as in a calcium-free medium (Fig. 4a) or in shaken culture (Fig. 4b), growth does not stop until the concentration of sugar in the medium falls below $0.05 \%$.

Discussion-Soon after conidiation begins in strains of Neurospora crassa which sporulate heavily, the growth of the mycelium is arrested and autolysis occurs. Simultaneously, large amounts of aerial hyphae and conidia are formed; these are sufficient to account for the steady increase in the total weight of the culture, in spite of the decreased weight of vegetative mycelium. These changes are accompanied by rapidly diminishing concentrations of trehalose in the mycelium of strain $69-1113 \mathrm{~A}$ and a slower decline in strain 89601A. But while the mycelium loses trehalose, conidia acquire amounts of this sugar which range from $5 \%$ of their dry weight on the 4th day of growth to $10 \%$ on the 10th. As the origin of conidial trehalose in unknown, it is not known whether it is formed de novo or whether it is translocated intact from the mycelium. In contrast to the situation in conidiating strains, trehalose continues to accumulate in the mycelium of aconidial strains until maximal growth is attained.

Trehalase activity in the mycelium of the conidiating strains tested increases greatly following the initiation of conidiation until by the 10th day of growth 10-fold greater activity occurs. Moreover, the activity of this enzyme is 10-fold greater in newly formed conidia than in the vegetative mycelium. As in the case of ascospores of this fungus, both trehalose and trehalase coexist in ungerminated conidia, and in fact the sugar increases in concentration while the conidia remain attached to the mycelium in strain 69-1113A. Whereas compartmentation of the sugar and its hydrolase may explain the failure of the enzyme to act upon its substrate in dormant ascospores, this explanation probably

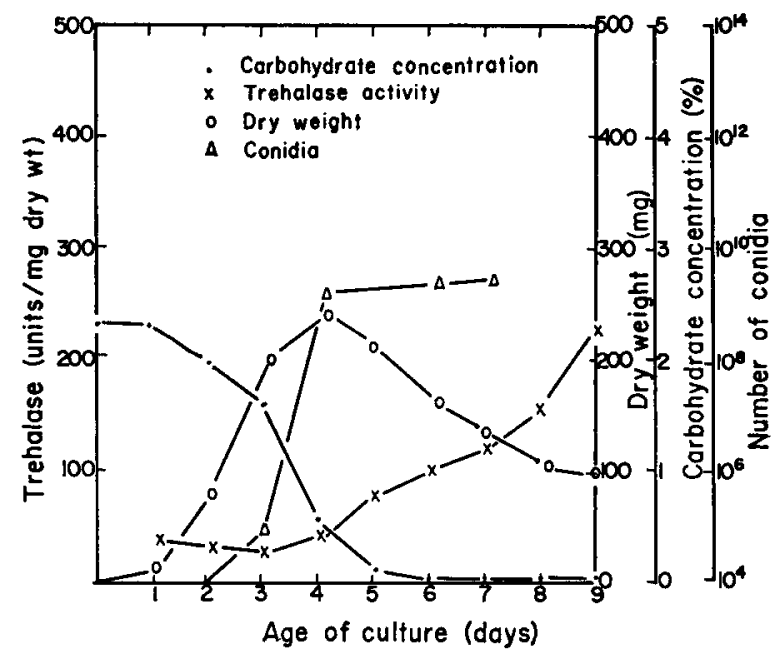

Fig. 6. Correlation between mycelial dry weight, mycelial trehalase activity, conidial production and the disappearance of sucrose from the growth medium of strain 69-1113A.

does not hold for conidia (Budd, Sussman, and Eilers, 1965).

However, trehalase activity in aconidial strains remains low until the end of the growth period. Moreover, the levels of trehalase activity in weakly conidiating strains are intermediate between those in aconidial and heavily conidiating ones. Some treatments which suppress conidiation also suppress trehalase activity, including growth in Turian's medium and in one that is calcium-free. Among the enzymes tested trehalase shows the greatest increase in activity when aconidial and conidial strains are compared.

The limited production of trehalase in aconidial strains and under conditions where conidiation is suppressed suggests that the formation of the enzyme might be closely associated with spore formation. However, the use of strain Y $843 \mathrm{~mA}$, in which conidiation can be successfully synchronized, led to the finding that the increase in trehalase activity in the mycelium lags behind conidiation. Thus, although trehalase is part of the complement of spore enzymes and increases in its activity accompany conidiation, no causal relation seems to exist between the two events. The increase in trehalase activity can be dissociated from conidiation in another way because large increases in its activity are obtained in shaken cultures, even in the absence of conidiation. It is likely that the hydrolase is necessary to the spore because of its high content of trehalose which serves as an important endogenous food reserve. Therefore, increases in trehalase activity must accompany conidiation, a process which requires the mobilization of considerable reserves.

Both conidiation and trehalase formation begin when growth slows down in cultures of Neuro- 
spora. Many observations of a similar kind in higher plants led Molisch (1938) to postulate that senescence in these organisms results from the pre-emption of metabolites by reproductive organs. Although this theory is not consistent with all the evidence from higher plants (cf. Leopold, 1967), it is of interest that the growth of conidiating cultures of Neurospora ceases before the carbohydrate concentration is fully depleted. Therefore, a mechanism exists in conidiating cultures to arrest growth before the medium is exhausted, thereby diverting the use of exogenous carbohydrates to the fruiting process.

Even relatively high levels of sugar in the growth medium have no deleterious effect upon the initiation of production of macroconidia in Neurospora crassa. Thus, under standard growth conditions, at the end of the 3rd day when the sugar concentration exceeds $1.5 \%$, $2.7 \times 10^{6}$ conidia per $\mathrm{ml}$ are formed. Moreover, approximately twice as many are produced in $6 \%$ sucrose as in $2 \%$ and the time when they are initiated is not altered. Furthermore, as the data in Table 3 establish, conidiation may commence in the presence of high sugar concentration in the growth medium. Here it was observed that when the initial level of sugar in the medium was $8.0 \%$, conidiation began in the presence of $7.33 \%$ sucrose. However, by contrast, trehalase formation is delayed in higher concentrations of sugar (Fig. 5b), thereby dissociating the two events in yet another way. The effect of carbo- hydrate concentration on trehalase formation led to the studies reported in the next paper in this series which deal with the mechanisne whereby the activity of this enzyme is contronat.

\section{LTERATURE CITED}

Budd, K., A. S. Sussman, and F. I. Eillers. 1965. Glucose-C ${ }^{14}$ metabolism of dormant and activated ascospores of Neurospora. J. Bact. 91: 551-561.

Halvorson, H. O. [ed.] 1962. Spores II. A symposium. Burgess Publishing Co., Minneapolis, Minn.

HAWKER, L. E. 1.957. The physiology of reproduction in fungi. Cambridge Univ. Press, Isondon.

Hill, E. P., and A. S. Sussman. 1963. Purification and properties of trehalase(s) from Neurospora. Arch. Biochem. Biophys. 102: 389-396.

$\longrightarrow$, and - 1964. Development of trehalase and invertase activity in Neurospora. J. Bact. 88: 1556-1566.

Lwopold, A. C. 1967. Developmental aspects of plant senescence, p. 285-290. Proc. 17th Int. Hort. Congr. Vol. 3.

Molisch, H. 1938. Der Einfluss einer Pflanze auf die Andere, p. 12. Allelopathie. G. Fischer, Jena.

Neison, N. 1944. A photometric adaptation of the Somogyi method for the determination of glucose. J. Biol. Chem. 153: 375-380.

Rowher, A. G., E. R. Henschel, and C. E. Engei. 1965. Determination of glucose in corn syrup and sugars. J. Ass. Offic. Agr. Chem. 48: 844-848.

Turian, G. 1964. Synthetic conidiogenous media for Neurospora crassa. Nature 202: 1240-1241.

VogEL, H. J. 1956. A convenient growth medium for Neurospora (medium N). Microbiol. Genet. Bull. 13: $42-43$. 